\title{
THE MULTIPLE LAG PROCESS AND ITS ESTIMATION
}

\author{
M. Kemal Sönmez John S. Baras* \\ Institute for Systems Research and Electrical Engineering Department \\ University of Maryland College Park, MD 20742
}

\begin{abstract}
We derive estimators for the multiple lag process, a generalization of the lag process, via spectral representations of stationary processes by complex random spectral measures. We present estimators of transfer functions for the multiple lag model with a given vector of lags and derive a multiple-lag (quadratic) coherence which can be maximized to choose the best vector of lags in the minimum mean squared error sense from a given set of lag vectors. We also demonstrate the estimation scheme by a simulation example and point out possible applications for the multiple-lag model in speech processing.
\end{abstract}

\section{INTRODUCTION}

Let $\left\{X_{t}, Y_{t}\right\}, t=0, \pm 1, \pm 2, \ldots$, be a zero mean, vector process stationary to the fourth order. The lag process is defined as follows [2]

$$
L_{u}(t) \equiv X_{t} X_{t+u}-R_{X X}(u)
$$

where $R_{X X}(u)$ is the covariance function of $X_{t}$ and the $\operatorname{lag} u$ is a fixed integer. Clearly, $\left\{L_{u}(t)\right\}$ is a zero-mean process. The multiple lag process is a natural extension of the lag process, i.e. a vector of lag processes

$$
\mathbf{L}_{\mathbf{u}}(t) \equiv\left\{L_{u}(t), u \in \mathbf{u}\right\}
$$

where $\mathbf{u} \equiv\left\{u_{1}, \ldots, u_{N}\right\}$ is a vector of lags in the set $\mathcal{U} \subset 2^{\mathbf{Z}}$. The model of interest for the (multiple) lag process is

$$
Y_{t}=\sum_{k=-\infty}^{\infty} a_{k} X_{t-k}+\sum_{u \in \mathbf{u}} \sum_{k=-\infty}^{\infty} b_{k}^{u} L_{u}(t-k)+\epsilon_{t}
$$

where $\left\{a_{k}\right\},\left\{b_{k}^{u}\right\}$ are absolutely summable and $\left\{\epsilon_{t}\right\}$ is a stationary noise process independent of $\left\{X_{t}\right\}$.

Research supported in part by NSF Grant NSFD CDR 8803012, through the Engineering Research Center Program and by a grant from Texas Instruments.

* Martin Marietta Chair in Systems Engineering
The problem of interest in this paper is the estimation of $\left\{a_{k}\right\},\left\{b_{k}^{u}\right\}$, and selection of $\mathbf{u}$ from a set of lag vectors $\mathcal{U}$. The main mathematical tool used throughout the analysis is the spectral representations of stationary processes. We outline the random spectral measures in Section 2 to establish notation, and give the derivation of the estimators for the single lag case in Section 3. The generalization to multiple lags is sketched in Section 4. Section 5 contains an example and discussion of several areas where the multiple lag process may be useful.

The multiple lag process has been introduced by Kedem in [2] where estimators have been derived using orthogonal Wiener filters. Currently, the authors of this paper are co-authoring a paper with B. Kedem [4] which discusses both approaches and presents the application of the multiple lag process to short term speech prediction.

\section{SPECTRAL REPRESENTATION VIA COMPLEX RANDOM MEASURES}

The Wiener-Khintchine theorem establishes the spectral theory of stationary processes by providing a Fourier representation for the autocovariance function. It is of interest to the development in this paper that a similar representation exists for the process itself in terms of a random spectral measure. In the following, $\xi_{X}(\cdot)$ refers to the random spectral measure of the process $X_{t}$ which is defined over Borel-subsets of $(-\pi, \pi]$ and assigns random, complex-valued weights to Borel-subsets. Thus, $\xi_{X}(A)$ is a complex-valued random variable. For two disjoint subsets $A$ and $B, \xi_{X}(A)$ and $\xi_{X}(B)$ are uncorrelated. There also exists a real-valued non-random measure $F(\cdot)$ such that

$$
E\left[\xi_{X}(A) \overline{\xi_{X}(B)}\right]=F(A \cap B)
$$

where $\overline{(\cdot)}$ is the complex conjugate.

A stochastic integral with respect to $\xi_{X}(\cdot)$ may be constructed by (i) defining the integral for complexvalued step functions defined over partitions of $(-\pi, \pi]$ 
as a weighted sum of random variables obtained from the partition by $\xi_{X}(\cdot)$ (ii) observing the limits in mean square of such sums as the partition gets finer [3]. Therefore, for random processes $X_{t}$ which are zero-mean and weakly stationary, it makes sense to speak of representations such as

$$
X_{t}=\int_{-\pi}^{\pi} e^{i t \omega} \xi_{X}(d \omega)
$$

which satisfy

$$
E\left[X_{s} \overline{Y_{t}}\right]=R_{X Y}(s-t)=\int_{-\pi}^{\pi} e^{i s \omega} e^{-i t \omega} f_{X Y}(\omega)(d \omega)
$$

Here, $f_{X Y}(\omega)$ is the cross-spectral density and $d \omega$ may be thought of as a small interval containing $\omega$. In the following sections, we make use of spectral representations extensively.

\section{SINGLE LAG PROCESS}

Let $A(\omega)\left[B_{u}(\omega)\right]=\sum_{k=-\infty}^{\infty} e^{-i k \omega} a_{k}\left[b_{k}^{u}\right],-\pi<\omega \leq \pi$ assuming $a_{k}, b_{k}$ are absolutely summable. To facilitate the argument, we define a process $\left\{Z_{u}(t)\right\}$ as follows:

$$
Z_{u}(t) \equiv \sum_{k} a_{k} X_{t-k}+\sum_{k} b_{k} L_{u}(t-k)
$$

To start with, $\left\{X_{t}\right\},\left\{L_{u}(t)\right\},\left\{Z_{u}(t)\right\}$ and $\left\{Y_{t}\right\}$ are all stationary processes, so they possess spectral representations. Therefore, with the notation of Section 2, the following holds

$$
Y_{i}=\int_{-\pi}^{\pi} e^{i t \omega} A(\omega) \xi_{X}(d \omega)+\int_{-\pi}^{\pi} e^{i t \omega} B(\omega) \xi_{L_{u}}(d \omega)+\epsilon_{t}
$$

or

$$
Y_{t}=\int_{-\pi}^{\pi} e^{i t \omega} \xi_{Z_{u}}(d \omega)+\epsilon_{t}
$$

with

$$
\xi_{Z_{u}}(d \omega)=A(\omega) \xi_{X}(d \omega)+B(\omega) \xi_{L_{u}}(d \omega)
$$

as the spectral measure for the process $\left\{Z_{u}(t)\right\}$.

\subsection{Estimation of Transfer Functions}

We would like to obtain expressions for $A(\omega)$ and $B(\omega)$ in terms of spectral densities. We can get two equations by multiplying (7); (i) with $X_{t}$, (ii) with $L_{u}(t)$, and taking the expectation. The following fact and its corollary are used in their derivation which consists of replacing the processes with their spectral representations and evaluating the integrals:

$$
E\left[\xi_{X}(d \lambda) \overline{\xi_{Y}(d \omega)}\right]=1_{[\lambda=\omega]} f_{X Y}(\omega) d \omega
$$

$$
f_{Y X}(\omega)=\overline{f_{X Y}(\omega)}
$$

The following linear system of equations is thus obtained:

$$
\begin{gathered}
f_{Y X}(\omega)=A(\omega) f_{X X}(\omega)+B(\omega) f_{L_{u} X}(\omega) \\
f_{Y L_{u}}(\omega)=A(\omega) f_{X L_{u}}(\omega)+B(\omega) f_{L_{u} L_{u}}(\omega)
\end{gathered}
$$

Solving the linear system, we find

$$
\begin{aligned}
& A(\omega)=\frac{f_{L_{u} L_{u}}(\omega) f_{Y X}(\omega)-f_{L_{u} X}(\omega) f_{Y L_{u}}(\omega)}{f_{L_{u} L_{u}}(\omega) f_{X X}(\omega)-\left|f_{L_{u} X}(\omega)\right|^{2}} \\
& B(\omega)=\frac{f_{X X}(\omega) f_{Y L_{u}}(\omega)-f_{X L_{u}}(\omega) f_{Y X}(\omega)}{f_{L_{u} L_{u}}(\omega) f_{X X}(\omega)-\left|f_{L_{u} X}(\omega)\right|^{2}}
\end{aligned}
$$

as estimators for transfer functions in terms of spectral densities.

\subsection{Lagged coherence}

Now, using the fact that $\epsilon_{t}$ is independent of $Z_{u}(t)$, we can sum the spectral densities to get

$$
f_{Y Y}(\omega)=f_{Z_{u} Z_{u}}(\omega)+f_{\epsilon \epsilon}(\omega)
$$

From (10), we write

$$
f_{Z_{u} Z_{u}}(\omega)=E\left[\xi_{Z_{u}}(d \omega) \overline{\left(\xi_{Z_{u}}(d \omega)\right)}\right]
$$

which because of (9) leads to

$$
\begin{aligned}
& f_{Z_{u} Z_{u}}(\omega)=|A(\omega)|^{2} E\left[\xi_{X}(d \omega) \overline{\xi_{X}(d \omega)}\right] \\
& \quad+|B(\omega)|^{2} E\left[\xi_{L_{u}}(d \omega) \overline{\xi_{L_{u}}(d \omega)}\right] \\
& \quad+2 \Re\left\{A(\omega) \overline{B(\omega)} E\left[\xi_{X}(d \omega) \overline{\xi_{L_{u}}(d \omega)}\right]\right\} .
\end{aligned}
$$

From (18) and (10) we conclude

$$
\begin{aligned}
& f_{Y Y}(\omega)=|A(\omega)|^{2} f_{X X}(\omega)+|B(\omega)|^{2} f_{L_{u} L_{u}}(\omega) \\
& \quad+2 \Re\left\{A(\omega) \overline{B(\omega)} f_{X L_{u}}(\omega)\right\}+f_{\epsilon \varepsilon}(\omega) .
\end{aligned}
$$

Rearranging, one gets

$$
f_{\epsilon \epsilon}(\omega)=f_{Y Y}(\omega)[1-\Gamma(\omega ; u)]
$$

where

$$
\begin{aligned}
\Gamma(\omega ; u) & \equiv \frac{f_{Z_{u} Z_{u}}(\omega)}{f_{Y Y}(\omega)} \\
= & \frac{1}{f_{Y Y}(\omega)}\left[|A(\omega)|^{2} f_{X X}(\omega)+|B(\omega)|^{2} f_{L_{u} L_{u}}(\omega)\right] \\
& +\frac{2}{f_{Y Y}(\omega)}\left[\Re\left\{A(\omega) \overline{B(\omega)} f_{X L_{u}}(\omega)\right\}\right]
\end{aligned}
$$

From (20), it is evident that

$$
0 \leq \Gamma(\omega ; u) \leq 1, \quad \forall \omega, u .
$$


If (7) is a good model for the given data series, the noise power must be small. Therefore, the best lag $u^{*}$ may be estimated by maximizing $\Gamma(\omega ; u)$ over $u$ since the particular model parameter $u^{*}$ which maximizes $\Gamma(\omega ; u)$, minimizes the noise variance as can be seen from (20):

$$
u^{*}=\arg \max _{u} \Gamma(\omega ; u) .
$$

Notice that $u^{*}$ is a function of $\omega$ and in general need not be unique over the whole frequency band.

\section{MULTIPLE LAG PROCESS}

The processes $\left\{X_{t}\right\}$ and $\left\{\mathbf{L}_{\mathbf{u}}(t)\right\}$ admit spectral representations which allow us to write $(2)$ as

$$
\begin{aligned}
Y_{t}= & \int_{-\pi}^{\pi} e^{i t \omega} A(\omega) \xi_{X}(d \omega) \\
& +\sum_{u \in \mathbf{U}} \int_{-\pi}^{\pi} e^{i t \omega} B_{u}(\omega) \xi_{L_{u}}(d \omega)+\epsilon_{t}
\end{aligned}
$$

where $\xi_{X}(\cdot)$ and $\xi_{L_{u}}(\cdot)$ are random spectral measures as defined in Section 2. The key to the development is the definition of the following spectral measure

$$
\xi_{Z_{u}}(d \omega) \equiv A(\omega) \xi_{X}(d \omega)+\sum_{u \in \mathbf{u}} B_{u}(\omega) \xi_{L_{u}}(d \omega)
$$

where, as in Section $3, Z_{u}(t)$ is defined to be:

$$
Z_{u}(t) \equiv \sum_{k} a_{k} X_{t-k}+\sum_{u \in \mathbf{u}} \sum_{k} b_{k}^{u} L_{u}(t-k) .
$$

\subsection{Estimation of Transfer Functions}

Now, by using properties of the random spectral measures we derive estimators for the linear and quadratic transfer functions $A(\omega), B_{u}(\omega)$ in terms of the spectral densities of the processes. We derive a linear system of equations for the the transfer functions of the model with the given set of lags $\mathbf{u}$ in terms of the spectral densities of the processes. Let us define the vector of transfer functions

$$
\mathbf{B}(\omega) \equiv\left(\begin{array}{c}
A(\omega) \\
B_{u_{1}}(\omega) \\
B_{u_{2}}(\omega) \\
\cdot \\
\cdot \\
B_{u_{N}}(\omega)
\end{array}\right)
$$

Extending the argument in Section 3, it can be shown that

$$
\mathbf{B}(\omega)=\mathbf{F}(\omega)^{-1} \mathbf{C}(\omega)
$$

where $\mathbf{C}(\omega)$ is the vector of cross-spectra between the lagged processes and the output

$$
\mathbf{C}(\omega) \equiv\left(\begin{array}{c}
f_{Y X}(\omega) \\
f_{Y X_{u_{1}}}(\omega) \\
f_{Y X_{u_{2}}}(\omega) \\
\cdot \\
\cdot \\
f_{Y X_{u_{N}}}(\omega)
\end{array}\right)
$$

and $\mathbf{F}(\omega)$ is a matrix containing cross-spectra of lagged processes

$$
\mathbf{F}_{i j}(\omega) \equiv f_{X_{u_{i}} X_{u_{j}}}(\omega), \quad i, j=0,1,2, \ldots, N
$$

where, for convenience, $X_{u_{0}} \equiv X$.

Thus, estimators of the transfer functions can be obtained as functions of spectral density estimators. Note that the introduction of the lag processes has transformed a nonlinear (quadratic) problem in $X_{t}$ to a linear problem in quadratic processes $L_{u}(t)$, thus allowing the solution to be expressed in terms of bispectra only.

\subsection{Multiple Lag Coherence:}

It can be shown in a similar way to that in Section 3 that the following quantity which we call multiple lag coherence must be maximized to estimate (choose) the optimum set of lags in the minimum mean square error sense.

$$
\begin{aligned}
& \Gamma_{\mathbf{u}}(\omega)=\frac{1}{f_{Y Y}(\omega)} \sum_{u=u_{0}}^{u_{N}}\left|B_{u}(\omega)\right|^{2} f_{L_{u} L_{u}}(\omega) \\
& +\frac{2}{f_{Y Y}(\omega)} \sum_{u \neq v} \Re\left\{B_{u}(\omega) \overline{B_{v}(\omega)}\right\} f_{L_{u} L_{v}}(\omega) \\
& -\pi<\omega \leq \pi
\end{aligned}
$$

where $B_{u_{0}}(\omega) \equiv A(\omega)$.

\section{SIMULATION EXAMPLE:}

A lag process with a lag of $u=2$ was synthesized using Gaussian noise as the input process $X_{t}$. The estimators for the transfer functions were computed using Bartlett window smoothed periodograms of the spectral densities for $u=0,1,2, \ldots, 10$. Figures 1 and 2 depict the values of the quadratic coherence in terms of $\omega$ and $u$ respectively. It is seen that there is a strong maximum at the true parameter $u=2$, and the rank holds throughout the whole spectrum.

In conclusion, the estimation of the process involves the estimation of transfer functions for the sets of lags in the lag set, and the maximization of the multiple lag coherence over the set. The analytic expressions for 
both the transfer function estimators and the quadratic coherence have been derived. In general, a unique set of lags which maximize the quadratic coherence throughout the spectrum does not have to exist and different sets of lags may maximize it over different bands. In practical applications such as speech processing, the set of lags which maximize the coherence over a perceptual sampling of the frequency band may be chosen.

The intended application for the nonlinear model is short-term prediction in speech coding, especially as the short-term predictor in set excited analysis by synthesis coders. Currently, such coders are based on linear models which do not allow extraction of non-linear dependencies, the existence of which has been demonstrated in [5].

\section{REFERENCES}

[1] Kedem, B. "An Orthogonal Representation of Nonlinear Systems", Carnegie-Mellon University Dept. of Statistics Tech. Rep. \#66, June 1972.

[2] Kedem-Kimelfeld, B., "Estimating the Lags of Lag Processes," Journal of the American Statistical Association, 70 (September 1975), 603-605.

[3] Kedem, B. Time Series Analysis by Higher Order Crossings, New York: IEEE Press, 1994.

[4] Sönmez, M.K., Kedem B., Baras, J., in preparation. [5] Thyssen, J., Nielsen, H., Hansen, S.D., "Non-linear Short Term Prediction in Speech Coding," ICASSP 94, Adelaide, Australia, I, 185-188.

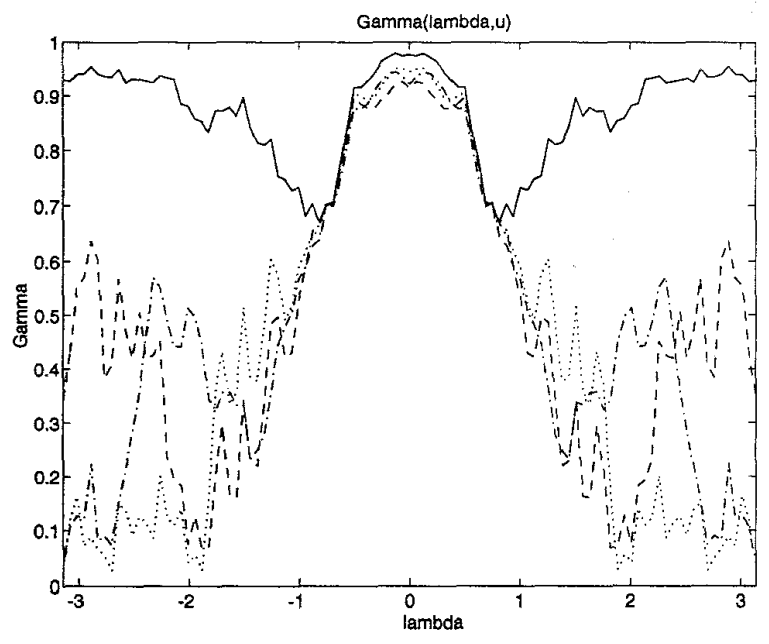

Figure 1: Quadratic coherence vs. frequency: $\Gamma(\omega ; 0)(--), \Gamma(\omega ; 1)(\cdots), \Gamma(\omega ; 2)($ solid $), \Gamma(\omega ; 3)(-\cdot-)$

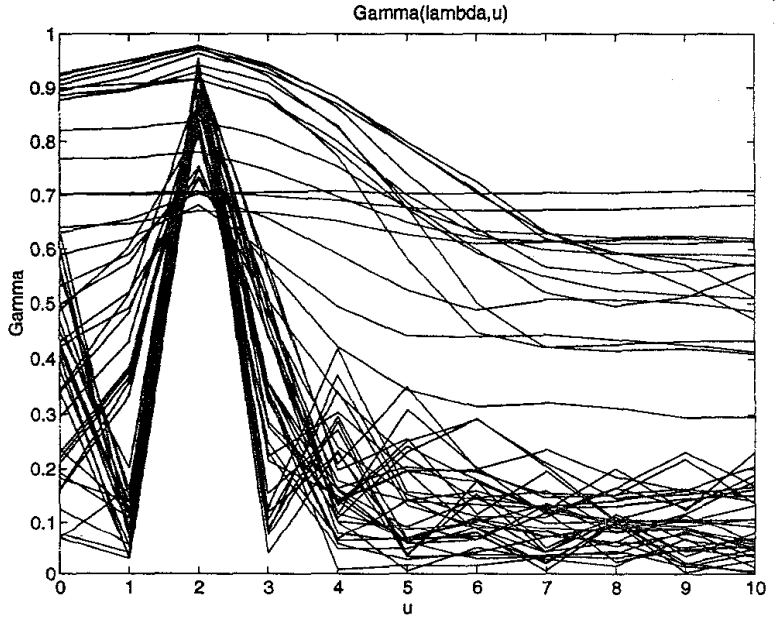

Figure 2: Quadratic coherence vs. delay: $\Gamma\left(0, \frac{\pi}{256}, \frac{2 \pi}{256}, \ldots, \frac{255 \pi}{256}, \pi ; u\right)$ 